\title{
A Comparison of Spectrophotometric and Oxidoreduction Potential Method for Laccase Activity Measurement
}

\author{
Rana Mustafa ${ }^{1 *}$, Duried Alwazeer ${ }^{2}$ and B Dave Oomah ${ }^{3}$ \\ ${ }^{1}$ Department of Plant Sciences, Canada \\ ${ }^{2}$ Center for Redox Applications in Foods (RCRAF), Turkey \\ ${ }^{3}$ Retired, Formerly with the National Bioproducts and Bioprocesses Program, Summerland, British Columbia, VOH 1Z0, Canada \\ *Corresponding author: Rana Mustafa, College of Agriculture and Bioresources, Department of Plant Sciences, College of Agriculture and Bio \\ resources, 51 Campus Drive, Saskatoon, SK S7N 5A8, Canada
}

Submission: 盎 October 06, 2017; Published: 眥 November 07, 2017

\begin{abstract}
Polyphenol oxidases (PPOs) oxidize phenolic compounds forming quinones, which undergo non-enzymatic reactions resulting in colored compounds. We studied ferulic acid and catechol oxidation by Myceliophthora thermophila laccases using oxidoreduction potential (ORP or Eh) as a novel method to determine PPO activity compared to the traditional spectrophotometric method. Eh varied time-dependently on ferulic acid and catechol concentrations. Eh increased to a maximum value ( $\mathrm{Eh}_{\mathrm{max}}$ ), then decreased irreversibly proportional with substrate concentration. The $\Delta$ Eh curves of ferulic acid and catechol oxidation at different concentrations by laccase exhibited two steps substrate oxidation: enzymatic and non-enzymatic; linear segments with increasing and decreasing slopes (rate of $\Delta \mathrm{Eh}$ change). The $\mathrm{K}_{\mathrm{m}}$ values determined spectrophotometrically and $0 \mathrm{RP}$ were 0.55 , 0.75 and $0.39,0.32 \mathrm{mM}$ for catechol and ferulic acid, respectively. The kinetic parameters $\left(\mathrm{K}_{\mathrm{m}}, \mathrm{V}_{\max }\right)$ of enzymatic reaction were not significantly different between the spectrophotometry and ORP methods. The ORP method is simple, low-cost and fast enabling the differentiation between the enzymatic and nonenzymatic phenol oxidation reactions.
\end{abstract}

Keywords: Myceliophthora thermophila laccases; Polyphenol oxidase; Enzyme kinetic; Oxidoreduction potential; Phenolic compound

Abbreviations: ADA: 4 Amino N, N-Diethyl Aniline Sulphate; KM: Michaels Menten Kinetics Constants; L-DOPA: L-3,4 Dihydroxyphenylalanine; LACs: Laccase; ORP: Oxidoreduction Potential; PPOs: Polyphenol Oxidizes; S: Substrate; V: Reaction velocity; Vmax: Maximum velocity

\section{Introduction}

Polyphenol oxidases (PPOs) are responsible for enzymatic browning during the postharvest steps of fruits and vegetables processing [1]. This reaction consists of two steps: enzymatic and non-enzymatic. In the enzymatic phase, PPOs oxidize the monophenols or o-diphenols in the presence of oxygen forming quinones that are unstable intermediate compounds. The quinones then no-enzymatically react with other compounds in the medium or polymerize to form melanins as the end products [2]. PPOs (1,2-benzenediol:oxygen oxidoreductase) are metalloproteins containing copper atoms in the active site and are divided into two groups catechol oxidize (EC 1.10.3.1) and laccases (EC 1.10.3.2) $[3,4]$. Laccases have broad substrate specificity and are able to transform a wide range of compounds. Considerable attention has been devoted to laccases as potential industrial enzymes in various applications, such as pulp delignification, wood fiber modification, dye or stain bleaching, chemical or medicinal synthesis, and contaminated water or soil remediation [5-8]. Furthermore, laccases can also be used in the food industry such as beverage (wine, fruit juice and beer) processing, sugar beet pectin gelatin, baking, food antioxidant and colorants synthesis $[9,10]$.

PPO activity is most commonly determined by the colorimetric methods using natural and synthetic substrates such as L-3,4 Dihydroxyphenylalanine (L-DOPA) and diazo derivatives of phenol. However, catechol is considered a standard substrate in the oxygen consumption method determined by oxygen concentration with an electrode in an oxygen saturated buffer [11]. Catechol as substrate was unable/ ineffective in differentiating PPO activity of forages determined spectrophotometrically by measuring the initial linear rate of change in absorption $(420 \mathrm{~nm}$, to measure quinones production prior to secondary complex formation). Earlier, PPO activity was determined by a chronometric method measuring the time required for supplemented ascorbic acid oxidation. In this system, PPO catalyzes the ascorbic acid to catechol to o-quinones, which in turn oxidizes ascorbic acid to dehydroascorbic acid and 
is reduced back to catechol [12]. A year later the same group using a polarized rotating platinum electrode enabled PPO activity measurement at various ascorbic acid concentrations continuously recording oxygen consumption [13]. Furthermore, the oxygenuptake curves were non-linear with time.

A photometric method $\left(\lambda 617 \mathrm{~nm}, \varepsilon=11,080 \mathrm{M}^{-1} \mathrm{~cm}^{-1}\right.$ in dichloromethane) has been proposed to measure PPO activity in olives in the presence of 4-amino-N, N-diethyl Aniline Sulphate (ADA) - excellent substrate for laccase and PPOs [14]. The same principle enabled the rapid detection of catecholase activity of PPO on slab gels based on 4-tert-butyl catechol oxidation to yellow o-quinones, which in turn quickly reacts with the coupling agent ADA leading to a deep blue adduct [15]. Other methods such as optical and amperometric biosensors and visible near-infrared hyper spectral imaging have also been used to predict PPO activity in a non-destructive manner [16]. Recently measured polyphenol oxidase activity using optical waveguide light mode spectroscopybased immunosensor. The sensor was suitable for detecting very low PPO activity that might be present in some food samples for which a conventional colorimetric assay may not be applicable.

Previously, we investigated ferulic acid oxidation by Myceliophthora thermophila laccase to synthesize 'nature identical' stable pigments that could be used in cosmetics and foods [10]. In aqueous medium, the rate of ferulic acid conversion (0$60 \mathrm{~min}$ ) decreased from $5-0.5 \mathrm{~mm}$ with negligible yield of yellow colour products, due to the higher activity of both enzyme and semi-quinones radical [10]. Recently, a purified Myceliophthora thermophila laccase was used as a biocatalyst to enzymatic ally oxidize ferulic acid in aqueous medium using an eco-friendly procedure to synthesize new active molecules [17]. Diametric species of ferulic acid (molecular mass $386 \mathrm{~g} / \mathrm{mol}$ ) were found after complete ferulic acid oxidation (150min) by the purified Myceliophthora thermophila laccase [17]. In this investigation, we studied ferulic acid and catechol oxidation by Myceliophthora thermophila laccase using oxide reduction potential as a novel method to determine PPO activity compared to the traditional spectrophotometric method.

\section{Materials and Methods}

\section{Sample preparation and chemicals}

Laccase $(1.15 \mathrm{~g} / \mathrm{ml})$ produced from Myceliophthora thermophila was obtained from Novozyme and ferulic acid was purchased from Fluka (Buchs, Germany) and catechol from Merck (Germany). Reaction medium was composed of phenol compound $(0.5,1,3,5$, $7 \mathrm{~mm}$ ) dissolved in $0.05 \mathrm{M}$ sodium phosphate buffer (pH 7.5) and laccase.

\section{Enzymatic activity determination by spectrophotometric method}

Laccase activity was assayed with catechol and ferulic acid as substrate. The increase in absorbance at $350 \mathrm{~nm}$ (ferulic acid) and $438 \mathrm{~nm}$ (catechol) at $30^{\circ} \mathrm{C}$ was measured with a spectrophotometer (Spectro UV-VIS Double Beam PC, 8 Scanning Auto Cell, Labored,
Inc. USA). For the enzymatic assays $1.5 \mathrm{ml}$ of the aqueous substrate stock solution $(10 \mathrm{~mm})$ was added to $1.48 \mathrm{ml}$ of phosphate buffer $(0.05 \mathrm{M}, \mathrm{pH} 7.5)$. The enzymatic reaction was initiated by adding $20 \mu \mathrm{L}$ of laccase. The blank sample containing all the components except the enzyme was performed at the same time. The initial reaction rates $\left(\mathrm{V}_{0}\right)$ were computed from the linear slopes of the absorbance-time curve of enzymatic step of oxidation reaction (within 2min).

\section{Enzymatic activity determination by oxidoreduction potential (ORP) method}

The oxidoreduction potential (ORP or Eh) of the reaction medium was measured with a combined electrode (SP50, Consort, Belgium) referred to the $\mathrm{Ag} / \mathrm{AgCl}$ system and connected to a redoxcontrolled interface (C835, Consort, Belgium). The DIS software was used to monitor the Eh and pH values of the reaction medium. The Eh values were plotted against time, and the initial reaction rates (V0) were calculated from the linear slopes of the Eh-time curve of the first part of the curve (from beginning of the reaction to the maximum Eh value).

\section{Determination of kinetic constants}

Laccase activities were measured with five different concentrations of each substrate $(0.5,1,3,5,7 \mathrm{~mm})$ to determine the Michaels-Menten kinetics constants $\left(\mathrm{K}_{\mathrm{m}}\right)$ and maximum velocity $\left(\mathrm{V}_{\max )}\right.$ values. Data were plotted as $1 /[\mathrm{V}]$ and $1 /[\mathrm{S}]$ for the Line weaver-Burk graphs [18].

\section{Results and Discussion}

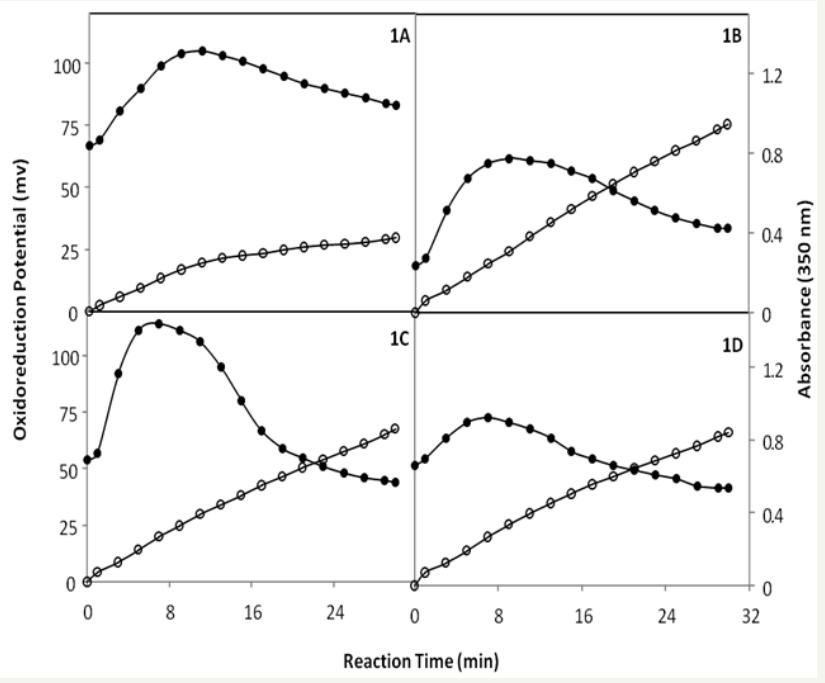

Figure 1: Absorbance (ABS) (o) and oxidoreduction potential $(\mathrm{Eh})$ values $(\bullet)$ for ferulic acid oxidation by laccase. Substrate concentrations: $0.5 \mathrm{~mm}(\mathrm{~A}), 3 \mathrm{~mm}$ (B), $5 \mathrm{~mm}$ (C) and $7 \mathrm{~mm}$ (D). The phenolic compound oxidation was determined spectrophotomically by the change in absorbance $(350 \mathrm{~nm}$ for ferulic acid) as well by determining the oxidoreduction potential (Eh) measured in MV, of the reaction medium. Experiments were carried out in triplicate, with relative standard deviation $>5 \%$.

(Figure 1) The absorbance $\left(\lambda_{\max }=350 \mathrm{~nm}\right)$ due to ferulic acid 
oxidation by Myceliophthora thermophila laccase increased linearly (r2= 0.99) with time (0-30min) for all concentrations except $0.5 \mathrm{~mm}$ (Figure $1 \mathrm{~A})$. At that low $(0.5 \mathrm{~mm})$ concentration, a parabolic equation $\left(\mathrm{Y}_{\mathrm{Abs}}[0.5 \mathrm{~mm}]=-0.0004 \mathrm{x}^{2}+0.0248 \mathrm{x}+0.0083 ; \mathrm{r}^{2}=0.9926\right)$ best described the increased absorbance with time indicating a two step process. This parabolic curve was similar to the previously reported relationship between absorbance variation at $310 \mathrm{~nm}$ and ferulic acid $(20-100 \mu \mathrm{M})$ consumption by wheat germ peroxides [19]. The saturation concentration of ferulic acid for laccase occurred at $0.6 \mathrm{~mm}[20]$ and may explain the behavior of ferulic acid oxidation at $0.5 \mathrm{~mm}$ observed in our study. The rate of absorbance increase with time was similar for 5 and $7 \mathrm{~mm}$ ferulic acid demonstrated by the same slope $(0.027 \mathrm{x})$. The oxidoreduction potential (Eh) varied with time depending on ferulic acid concentration. Thus, two linear segments (increase $0-9 \mathrm{~min}$ followed by a decrease 11$30 \mathrm{~min}$ ) occurred at low ferulic acid concentrations ( 0.5 and $3 \mathrm{~mm})$. At high concentrations ( 5 and $7 \mathrm{~mm}$ ), Eh values increased initially (0-7 min) followed by two step linear decrease from 9-21 min and 23-30min. Moreover, the plots for absorbance and oxidoreduction potential intercepted at 19, 23 and $21 \mathrm{~min}$ for 3,5 and $7 \mathrm{~mm}$ ferulic acid concentrations, respectively, perhaps indicating the end of the enzymatic reaction.

When catechol was used as substrate, absorbance values $\left(\lambda_{\max }=438 \mathrm{~nm}\right)$ always increased linearly $\left(\mathrm{r}^{2}=0.99\right)$ with time (3-31min) at all concentrations (Figure 1B). This linear rate of absorbance increase with time (a measure of quinones production prior to secondary complex formation) was similar for 1 and $5 \mathrm{~mm}$ catechol concentration with the same slope $(0.015 \mathrm{x})$ and parallel to the $7 \mathrm{~mm}$ catechol concentration. The highest and lowest slopes for absorbance as a function of time occurred at 0.5 and $3 \mathrm{~mm}$ catechol concentrations, respectively. Eh values showed a two steps process increasing then decreasing thereafter with time (3-31 min) thereby corresponding to parabolic equations for $0.5,3$ and $7 \mathrm{~mm}$ catechol concentrations. For $0.5 \mathrm{~mm}$ catechol, the inflection point occurred at $17 \mathrm{~min}$ resulting in two linear rates (increase from $3-17 \mathrm{~min}$ and decreasing thereafter $17-31 \mathrm{~min}$ ). This inflection point was observed at $13 \mathrm{~min}$ for $3 \mathrm{~mm}$ catechol for two linear rates $3-13 \mathrm{~min}$ (constant/unchanged) and 15-31min (decrease); $15 \mathrm{~min}$ for $7 \mathrm{~mm}$ catechol corresponding to two linear rates $3-15 \mathrm{~min}$ and $17-31 \mathrm{~min}$. These inflection points probably relate to the end of the enzymatic and the beginning of the non-enzymatic reactions.

The values of oxidoreduction potential of reaction medium increased with time attaining a maximum value $\left(E h_{\text {max }}\right)$, then decreased irreversibly proportional with substrate concentration. $E h_{\text {max }}$ values were obtained after $t=700,650,580$ and $410 \mathrm{sec}$ for $0.5,3,5$ and $7 \mathrm{~mm}$ ferulic acid, respectively (Figure $1 \mathrm{C}$ ).

The $\Delta$ Eh curves of ferulic acid and catechol oxidation at different concentrations by laccase exhibited two steps substrate oxidation: enzymatic and non-enzymatic (Figure 1D \& Figure 2). These two steps ferulic acid oxidation (Figure 2A) constituted two linear segments with increasing $\left(r^{2} \geq 0.95\right)$ and decreasing $\left(r^{2} \geq\right.$ 0.98 ) slopes (rate of $\Delta$ Eh change). At both low (0.5mm, 1-9min) and high (7mm, 1-5min) ferulic acid concentrations the increase in $\Delta \mathrm{Eh}$ was similar, whereas the slope for $5 \mathrm{~mm}(1-5 \mathrm{~min})$ was twice that of $3 \mathrm{~mm}(1-7 \mathrm{~min})$, indicating a doubling of the rate of $\Delta \mathrm{Eh}$ increase. The inflection point (maximum) for $\Delta$ Eh oxidation occurred at 11 , 9, 7 and $7 \mathrm{~min}$ for $0.5,3,5$ and $7 \mathrm{~mm}$ ferulic acid, respectively. The linear decrease (11-30min) in $\Delta \mathrm{Eh}$ was similar for 0.5 and $3 \mathrm{~mm}$ ferulic acid oxidation based on their slopes. The greatest reduction (steepest slope $11-19 \mathrm{~min}[-6.1 \mathrm{x}]$ ) in $\Delta \mathrm{Eh}$ was observed at $5 \mathrm{~mm}$ ferulic acid.

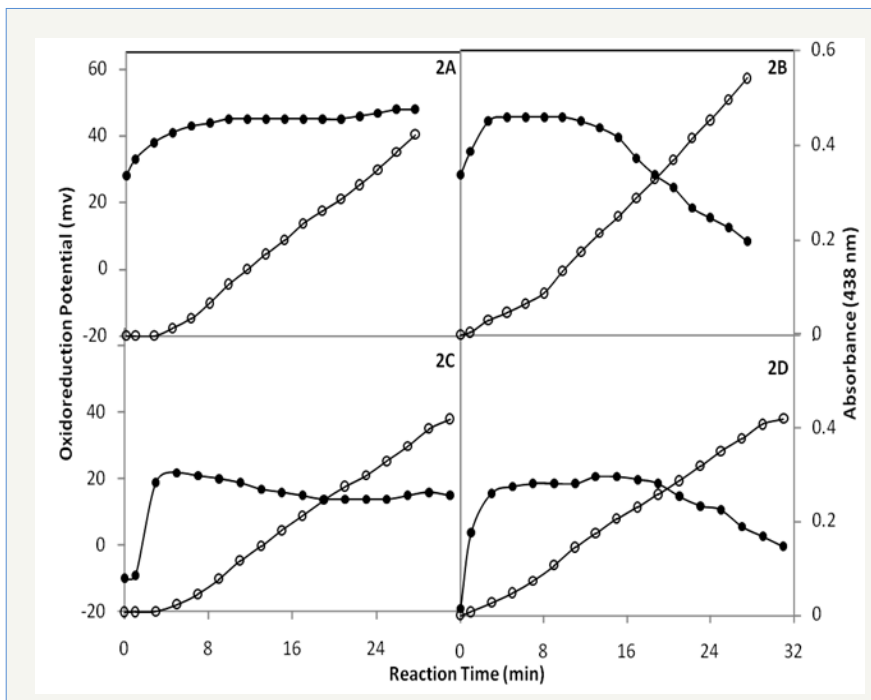

Figure 2: Absorbance (ABS) (o) and oxidoreduction potential $(\mathrm{Eh})$ values $(\bullet)$ for catechol oxidation by laccase. Substrate concentrations: $0.5 \mathrm{~mm} \mathrm{(A),} 3 \mathrm{~mm}$ (B), $5 \mathrm{~mm} \mathrm{(C)} \mathrm{and} 7 \mathrm{~mm}$ (D). The phenol compound oxidation was determined spectrophotomically by the change in absorbance (438 for catechol) as well by determining the oxidoreduction potential (Eh) measured in MV, of the reaction medium. Experiments were carried out in triplicate, with relative standard deviation $>5 \%$.

The variation in $\Delta \mathrm{Eh}$ with time (3-31min) fitted the parabolic equation $\left(\mathrm{r}^{2} \geq 0.80\right)$ for catechol oxidation by laccase at all concentrations (Figure 2B) indicating two steps process. However, linear segments were encountered for all catechol concentrations; $0.5 \mathrm{~mm}\left(1-19 \mathrm{~min} ; \mathrm{r}^{2}=0.96\right), 1 \mathrm{~mm}(7-32 \mathrm{~min} ; \mathrm{r} 2=0.81), 3 \mathrm{~mm}$ (13$\left.31 \mathrm{~min} ; \mathrm{r}^{2}=0.99\right), 5 \mathrm{~mm}\left(5-19 ; \mathrm{r}^{2}=0.99\right.$ and $\left.21-31 \mathrm{~min} ; \mathrm{r}^{2}=0.62\right)$ and $7 \mathrm{~mm}\left(3-17 \mathrm{~min} ; \mathrm{r}^{2}=0.76\right.$, and $\left.19-31 \mathrm{~min} ; \mathrm{r}^{2}=0.99\right)$. The linear segments indicated reductions in $\Delta \mathrm{Eh}$ values for 3 and $5 \mathrm{~mm}$ catechol due to their negative slopes.

Furthermore, absorbance values were inversely correlated with Eh for 5 and $7 \mathrm{~mm}$ ferulic acid oxidations ( $\mathrm{r}=-0.57$; $\mathrm{P}=0.0166$ and $\mathrm{r}=-$ $0.68 ; \mathrm{P}=0.0029$, respectively). These correlations were positive for 0.5 and $1 \mathrm{~mm}$ catechol $(\mathrm{r}=0.896 ; \mathrm{P}<0.0001$ and $\mathrm{r}=0.747 ; \mathrm{P}=0.0006$, respectively) and negative for $3 \mathrm{~mm}$ catechol $(\mathrm{r}=-0.831 ; \mathrm{P}<0.0001)$. The highly significant positive correlation between absorbance and Eh of catechol ( 0.5 and $1 \mathrm{~mm})$ was similar to those reported between the spectrophoto metric and oxygen consumption methods ( $\mathrm{r}=0.708$; $\mathrm{P}=0.033$ ) (Figure 2C) used to measure PPO activity of whole wheat with catechol as substrate [11].

On the other hand, using the Eh curves, the reaction rates were plotted against substrate concentration and the Kinetic constants 
were determined from Line weaver-Burk plot. The results were compared with those obtained from absorbance curves (spectrophotometrically method) and showed similar $\mathrm{K}_{\mathrm{m}}$ values referred to the affinity of enzyme to substrate (Table 1). The $\mathrm{K}_{\mathrm{m}}$ values determined spectrophotometrically and by oxidoreduction potential method were $0.55,0.75$ and $0.39,0.32 \mathrm{~mm}$ for catechol and ferulic acid, respectively. The differences in $V_{\max }$ values of the two methods may be attributed to the maximum reaction rate calculated for the whole reaction of substrate oxidation (enzymatic and non-enzymatic steps) in absorbance measurement (spectrophotometrically method), but was related only to the enzymatic reaction for the ORP method.

Table 1: Kinetic parameters of laccases biocatalysts determined by spectrophotometrically and oxidoreduction potential method.

\begin{tabular}{|c|c|}
\hline Subject Area & Food Chemistry and Analysis \\
\hline More Specific Subject Area & Polyphenol oxidize activity \\
\hline Type of Data & Table, figure, text file \\
\hline How Data was Acquired & $\begin{array}{c}\text { Spectrophotometer and combined electrode referred to the } \mathrm{Ag} / \mathrm{AgCl} \text { system and connected to a redox- } \\
\text { controlled interface }\end{array}$ \\
\hline Data Format & Raw, analyzed \\
\hline Experimental Factors & Eh and color of reaction medium was monitored during oxidation by laccases \\
\hline Experimental Features & $\begin{array}{l}\text { Myceliophthora thermophila laccases activity was assayed with catechol and ferulic acid as substrate. The Eh } \\
\text { values and the absorbance of the reaction medium were plotted against reaction time, and the initial reaction } \\
\text { rates (V0) were calculated from the linear slopes of the curve. }\end{array}$ \\
\hline Data Source Location & Homs (Syria), Saskatoon (Canada) \\
\hline Data Accessibility & Data is provided in the paper \\
\hline
\end{tabular}

The Michaels constant $\mathrm{K}_{\mathrm{m}}$ for ferulic acid (Table 1) was almost a quarter $(24 \%)$ that of a purified Myceliophthora thermophila laccase [17] and one order of magnitude lower than the ferulic acid oxidation by laccase from Coriolus hirsutus or Cerreba maxima [21]. The $\mathrm{K}_{\mathrm{m}}$ for catechol was over half (52\%) and only $16 \%$ those of laccases from Chaetomiacea and Fusarium solani, respectively [22]. Similar significant differences have been reported in kinetic parameters of different laccases with the same substrates [23].

The enzymatic oxidation of phenolic substrate by laccase occurred in two steps: enzymatic and non-enzymatic reactions. In the first step (enzymatic reaction) the enzyme oxidizes the phenolic substrate and forms colored intermediate products (semi quinones) which increase with time. These semi quinones are unstable compounds and have high oxidizing properties consequently the Eh of the medium attains a maximum value upon complete formation of semi quinones indicating the end of the enzymatic step of phenol compound oxidation. In the second step (non-enzymatic reactions) the semi quinones undergo condensation and polymerization which result in insoluble dark brown polymers known as melanins. Thus, the absorbance values of reaction medium increase with Eh reduction caused by the formation of the stable and low oxidizing compounds (melanins).

Laccases contain three types (T1,T2 and T3) Cu centers. The T2 and T3 sites form a pronuclear $\mathrm{Cu}$ cluster onto which $\mathrm{O}_{2}$ is reduced. The $\mathrm{T} 1 \mathrm{Cu}$ oxidizes the reducing substrate and transfers electrons to the $\mathrm{T} 2$ and $\mathrm{T} 3 \mathrm{Cu}$ [24]. The enzymatic oxidation of phenolic compounds by PPOs depends on oxidoreduction potential (Eh) of both enzyme and substrate. Some studies demonstrate a relationship between PPO activity and $\triangle \mathrm{Eh}$ between enzyme (especially T1) and substrate, where the enzyme can only oxidize the phenol substrates with Eh lower than that of the enzyme [24,25].
Furthermore, the rate of electrons transfer is related to $\Delta \mathrm{Eh}$; the Eh of substrate increases with reduction in the rate of electron transfer (from substrate to enzyme). Thus, the oxidation rate is faster at the higher Eh (laccase) or the lower Eh (phenol substrate) [26,27].

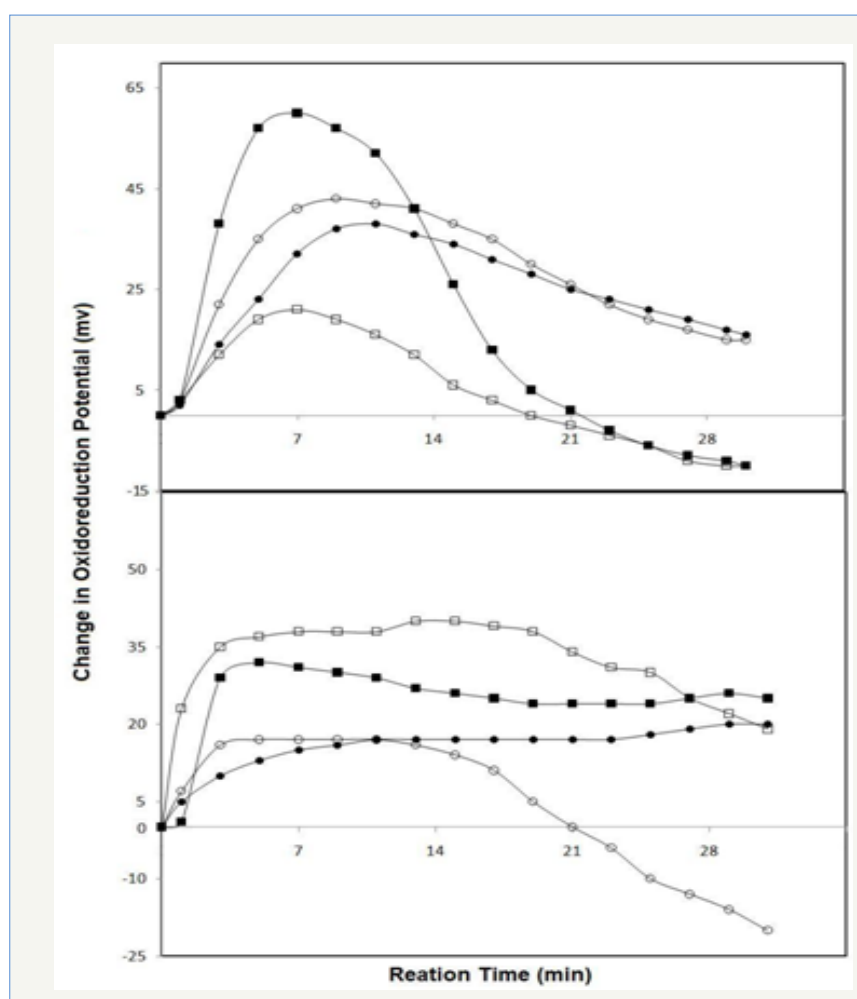

Figure 3: Change in oxidoreduction potential $(\Delta \mathrm{Eh})$ measured in MV, for ferulic acid (A) and catechol (B) oxidation by laccase, using different phenol acid concentration: $0.5 \mathrm{~mm}$ $(\bullet), 3 \mathrm{~mm}($ (о), $5 \mathrm{~mm}(\square)$ and $7 \mathrm{~mm}(\square)$. 
The Eh curves of ferulic acid and catechol oxidation at different concentrations $(0.5,3,5$ and $7 \mathrm{~mm})$ by laccase exhibited two steps of substrate oxidation: enzymatic and non-enzymatic (Figure 2D). Thus, the Eh reaction curve can be proposed to distinguish between the two steps of phenol oxidation by PPOs, which was not possible to carry out using classical methods such as the spectrophotometric method (Figure 3).

\section{Conclusion}

The results demonstrated the possibility of determining the activity of PPOs, such as laccase, by continuous monitoring the kinetic assays of oxidoreduction potential. The most important finding in this study was the possibility to distinguish between the enzymatic and non-enzymatic steps of phenol oxidation reaction, which was not possible to date by other techniques. This permitted also to control the work of the enzyme and separate quinones that possess attractive colors, antioxidant power and many industrial applications. The simplicity, low costs, and rapidity of the novel method can permit the researchers to carry out the experiments availably with easy tools.

\section{References}

1. Patel SS, Parekh SN (2015) Laccase-Production and its Application. World Journal of Pharmacy and Biotechnology 2(1): 57-63.

2. Whitaker JR, Lee CY (1995) Recent advances in chemistry of enzymatic browning. In Enzymatic Browning and Its Prevention American Chemical Society, Symposium Series 600, Washington DC, USA, pp. 2-7.

3. Mayer M (2006) Polyphenol oxidases in plants and fungi: Going places? A review. Phytochem 67(21): 2318-2331.

4. Sullivan ML (2015) Beyond brown: polyphenol oxidases as enzymes of plant specialized metabolism. Front Plant Sci 5: 783.

5. Ridgway T, Tucker G, Wiseman H (1997) Novel bioconversions for the production of designer antioxidant and colorant flavonoids using polyphenol oxidases. Biotechnol Genetic Engineer Rev 14: 165-190.

6. Figueroa EMC, Morel MH, Surget A, Rouau X (1999) Oxidative crosslinking of wheat arabinoxylans by manganese peroxidase. Comparison with laccase and horseradish peroxidase. Effect of cycteine and tyrosine on gelation. J Sci Food Agri 79(3): 460-463.

7. Mayer M, Staples C (2002) Laccase: new functions for an old enzyme. Phytochem 60(6): 551-565.

8. Zhanga J, Xu Z, Chen H, Zong Y (2009) Removal of 2,4-dichlorophenol by chitosan-immobilized laccase from Coriolus versicolor. Biochem Engineer Journal 45(1): 54-59.

9. Minussi RC, Pastore GM, Duran N (2002) Potential applications of laccase in the food industry. Trends in Food Science and Technology 13(6-7): 205-216.

10. Mustafa R, Muniglia L, Rovel B, Girardin M (2005) Phenolic colorants obtained by enzymatic synthesis using a fungal laccase in a hydroorganic biphasic system. Food Research International 38(8-9): 9951000 .

11. Vázquez D (2000) Effects of genotype and environment on polyphenol oxidase activity and related properties of red and white wheats. MSc. Thes is University of Manitoba Winnipeg MB, Canada, p. 173.

12. Ingraham LL, Makeover B (1955) Chronometric method of determining polyphenol oxidase activity. Potentiometric device for automatic determination of end point. Analytical Chemistry 27(6): 916-918.

13. Ingram LL (1956) Determination of polyphenol oxidase activity by rotating platinum electrode. Analytical Chemistry 28(7): 1177-1179.

14. Valgimigli L, Sanjust E, Curreli N, Rinaldi A, Pedulli GF, et al. (2001) Photometric assay for polyphenol oxidase activity in olives, olive pastes, and virgin olive oils. Journal of the American Oil Chemists' Society 78(12): 1245-1248.

15. Rescigno A, Sollai F, Rinaldi AC, Soddu G, Sanjust E, et al. (1997) Polyphenol oxidase activity staining in polyacrylamide electrophoresis gels. Journal of Biochemical and Biophysical Methods 34(2): 155-159.

16. Kim N, Kim WY (2015) Measurement of polyphenol oxidase activity using optical waveguide lightmode spectroscopy-based immunosensor. Food Chem 169: 211-217.

17. Aljawish A, Chevalot I, Jasniewski J, Paris C, Scher J, et al. (2014) Laccasecatalyzed oxidation of ferulic acid and ethyl ferulate in aqueous medium: A green procedure for synthesis of new compounds. Food Chem 145: 1046-1054.

18. Lineweaver H, Burk D (1934) The determination of enzyme dissociation constants. Journal of the American Chemical Society 56(3): 666-658.

19. Garcia R, Rakotozafy L, Telef N, Potus J, Nicolas J, et al. (2002) Oxidation of ferulic acid or arabinose-esterified ferulic acid by wheat germ peroxidises. J Agric Food Chem 50: 3290-3298.

20. Caunchio F, Crescenzi C, Girelli AM, Messina A, Tarola AM, et al. (2001) Oxidation of ferulic acid by laccase: identification of the products and inhibitory effects of some dipeptides. Talanta 55(1): 189-200.

21. Smirnov SA, Koroleva OV, Gavrilova VP, Belova AB, Klyachko NL, et al. (2001) Laccases from basidiomycetes: Physicochemical characteristics and substrate specificity towards methoxyphenolic compounds. Biochemistry (Moscow) 66(7): 774-779.

22. Lahtinen M (2013) Reactivity and reactions of lignin model compounds with laccases. PhD Thesis, (Academic Dissertation), University of Helsinki, Finland.

23. Viswanath B, Rajesh B, Janardhan A, Kumar AP, Narasimha G, et al. (2014) Fungal laccases and their applications in bioremediation. Enzyme Research, p. 21.

24. Xu F, Kulys JJ, Duke K, Li K, Krikstopaitis K, et al. (2000) Redox chemistry in laccase-catalyzed oxidation of N-hydroxy compounds. Appl Environ Microbiol 66(5): 2052-2056.

25. Xu F, Shin W, Brown SH, Wahleithner JA, Sundaram UM, et al. (1996) A study of a series of recombinant fungal laccases and bilirubin oxidase that exhibit significant differences in redox potential, substrate specificity, and stability. Biochimica et Biophysica Acta (BBA) - Protein Structure and Molecular Enzymology 1292(2): 303-311.

26. Cabiddu A, Lee MRF, Decandia M, Molle G, Salis L, et al. (2013) Characterization of polyphenol oxidase activity in a range of forage ecotypes with different phenol substrates. A new insight for PPO and protein-bound phenol evaluation. Grass and Forage Science 69: 678-692.

27. Pourcel L, Routaboul JM, Cheynier V, Lepiniec L, Debeaujon I, et al. (2006) Flavonoid oxidation in plants: from biochemical properties to physiological functions. Trends Plant Sci 12(1): 29-36. 